\title{
Periodontitis as a reservoir of human papillomavirus in the causation of oral squamous cell carcinoma: a review
}

\author{
Akanksha Raj, Neetha J. Shetty \\ Department of Periodontics, Manipal College of Dental Sciences, Mangalore at Manipal Academy of Higher Education, Karnataka, India
}

\begin{abstract}
Human papillomavirus (HPV) is a DNA virus from Papillomavirus family, and is one of the most widely reported sexual infection, which is not only related to ano-genital malignancies, but also associated with head and neck cancers, with chronic periodontitis being one of the risk factor for the same. Chronic periodontitis leads to loss of tissue structures of and around periodontium and is clinically detectable in the form of periodontal pocket and loss of alveolar bone. A variety of other systemic diseases have also been found to be linked to chronic periodontitis, such as cardiovascular diseases, respiratory diseases, etc., and recent data show that it is also related to malignancies of oral cavity. Junctional epithelium of periodontal pocket helps HPV to survive by facilitating the cellular functions to grow and hence, acts as a reservoir for the virus. Porphyromonas gingivalis, a Gram-negative anaerobic bacteria and HPV interacts both directly and indirectly in a series of complex reactions and as a result of some inflammatory reactions, this association further leads to commencement and progression of head and neck squamous cell carcinoma or oral squamous cell carcinoma. This article reviews the pathophysiology of oral human papillomavirus infection and its role in head and neck cancers, with chronic periodontitis as one of the causative factors.
\end{abstract}

HIV AIDS Rev 2020; 19, 2: 74-77

DOI: https://doi.org/10.5114/hivar.2020.96430

Key words: human papillomavirus, chronic periodontitis, Porphyromonas gingivalis, oral squamous cell carcinoma.

\section{Introduction}

Epidemiological data reveals human papillomavirus (HPV) as one of the most widely reported sexually transmitted infection worldwide [1]. It not only leads to anal and genital cancer but has an active role in etiopathogenesis of several oral malignancies [2,3]. Certain points of evidence support that uterine cervical epithelium and junctional epithelium of periodontal pocket shares some similarity in terms of their rapidly dividing basal layer, which provides greater affinity to HPV [4]. This indicates that chronic inflammation of periodontium that facilitates HPV life cycle may play a pivotal role in increasing the risk of oral cavity cancer. This review intends to explain the basic structure of $\mathrm{HPV}$ virus and its possible relation between periodontitis, which acts as a reservoir for the virus and helps in the progression of oral cavity carcinoma.

\section{Human papillomavirus structure}

HPV belongs to category of DNA viruses of family Papovaviridae, including Polyomaviruses and Papillomaviridae [5]. It is a non-enveloped virus having icosahedral capsid with double stranded DNA, and as it is a genetic material, is circular in shape [5].
International Journal of HIV-Related Problems

HIV \& AIDS R e v i e w 


\section{Human papillomavirus classification}

Phylogenetically, HPV can be classified into genera, species, and types [5]. Based on tropism, it can be further categorized as cutaneous type, causing lesions of skin and mucosal type, which is associated with anogenital and upper aerodigestive tract, initiating oral cancer [5]. HPV can also be classified based on oncogenicity [5].

\section{Human papillomavirus and chronic periodontal disease}

Periodontitis is a local inflammatory but chronic disease of oral cavity that is related to Gram-negative anaerobic bacteria present in dental plaque or biofilm, which damage periodontal tissues, and are clinically seen as periodontal pocket and loss of alveolar bone [6, 7]. HPV gains its entry either through a breach in mucosa or through normal exposure of parabasal cells, exclusively infecting basal cells of epithelium. In addition, the replication of HPV is closely related to proliferation of epithelial basal cell layer and parabasal cell layer. Initial HPV infection occurs in periodontal pocket, which consists of stratified squamous epithelium, and is characterized by continuous epithelial proliferation, migration, rete ridge formation, and ulcerations [8]. The bottom layer of periodontal pocket, i.e. the junctional epithelium, consists of suprabasal and basal cell layers only, have a tremendously high turnover ratio, and provides cellular functions required by the virus. Therefore, in a way, the periodontal pocket serves as a reservoir or tank for latent HPV that requires infected and undifferentiated cells for its survival. Before the process of differentiation, the basal cells become exfoliated from the gingival crevice [9].

\section{Association between chronic periodontal disease and oral cancer}

A close association between chronic periodontitis and oral cancer have been reported in literature. More than a four-fold increase in the risk of head and neck cancers has been reported with each millimeter loss of alveolar bone [10].

\section{Interaction of Porphyromonas gingivalis and human papillomavirus in the development and progression of oral carcinoma}

An interaction between oral bacterium, especially Porphyromonas gingivalis, and viruses occur either directly or indirectly. Lipopolysaccharides in the form of endotoxins, lytic enzymes like proteases, collagenases etc., and metabolic products in the form of hydrogen sulphide, ammonia etc., may directly carry out or induce changes at the genic level in adjacent cellular medium [11-13]. Additionally, it leads to an increased production of some substances like acetaldehyde [14-16] and nitrosamines $[17,18]$, which are quite carcinogenic in nature. Inflammatory reactions, on the other hand, explains the indirect association between these two. Neutrophils, lymphocytes, macrophages, fibroblasts, and epithelial cells of the host immune system respond to $P$. gingivalis by generating inflammatory mediators including: 1. Cytokines, chemokines, prostaglandins, growth factors, and other signals that provide an environment for various cellular functions like their survival, ability to proliferate, to migrate, to form new blood vessels (i.e. angiogenesis), and inhibition of apoptosis such as programmed cell death [19]. This new environment further

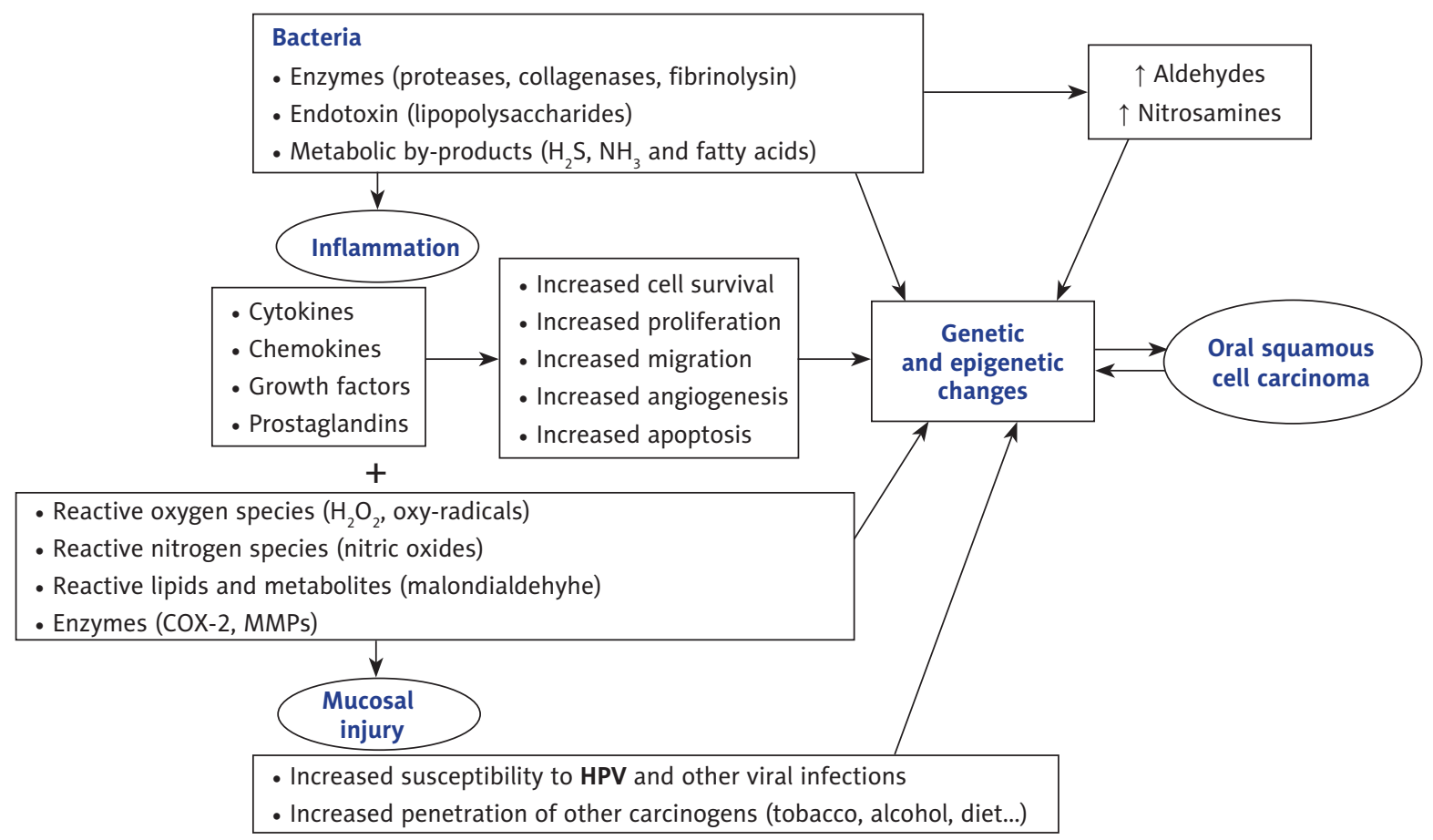

Figure 1. Inter-relationship between chronic periodontitis, HPV and oral squamous cell carcinoma [33] 
accumulates mutations in epithelial cells, and forces these mutant epithelial cells to migrate and proliferate at distant site and further allows them to grow; 2 . Along with inflammatory reactions, few other substances can also bring genetic and epigenetic changes by acting as endogenous mutagens. These substances include reactive oxygen species $\left(\mathrm{H}_{2} \mathrm{O}_{2}\right.$, oxy-radicals), reactive nitrogen species in the form of nitric oxides, reactive lipids, and few metabolites such as malondialdehyde and matrix-metalloproteinases [20-30]. Chronic inflammation may cause a breach in the mucosal barrier, which may further lead to an increased penetration of other carcinogenic substances like alcohol, tobacco, and dietary metabolites $[31,32]$.

However, it is yet determined whether it is the direct effect of bacteria or its indirect effect through stimulation of inflammation that links oral HPV infection with chronic periodontitis. Both the mechanisms are likely to be involved, but the majority of present data suggests that the periodontopathogenic bacterium travels via salivary pathway and bloodstream from affected sites to distant normal sites and carry out tissue injury through inflammatory reactions.

\section{Conclusions}

As literature has shown, chronic periodontitis is associated with various systemic diseases and other complications of oral cavity, such as oral carcinoma. This can act as an independent causative factor or in conjugation with HPV virus for the progression of oral carcinoma. Therefore, the management of chronic periodontitis patients becomes crucial in the maintenance of oral hygiene and health in order to prevent such potential life-threatening diseases like oral cancer.

\section{Conflict of interest}

The authors declare no conflict of interest with respect to the research, authorship, and/or publication of this article.

\section{References}

1. Suarez TP, Kelly JA, Pinkerton SD, et al. Influence of a partner's HIV serostatus, use of highly active antiretroviral therapy, and viral load on perceptions of sexual risk behavior in a community sample of men who have sex with men. J Acquir Immune Defic Syndr 2001; 28: 471-477.

2. Gillison ML, Koch WM, Capone RB, et al. Evidence for a causal association between human papillomavirus and a subset of head and neck cancers. J Natl Cancer Inst 2000; 92: 709-720.

3. Chaturvedi AK, Engels EA, Pfeiffer RM, et al. Human papillomavirus and rising oropharyngeal cancer incidence in the United States. J Clin Oncol 2011; 29: 4294-4301.

4. Nielsen FL (ed.). Progress in Oral Cancer Research. New York: Nova Medical Books; 2008. p. 98.

5. Kim SM. Human papillomavirus in oral cancer. J Korean Assoc Oral Maxillofac Surg 2016; 42: 327-336.

6. Loesche WJ, Grossman NS. Periodontal disease as a specific, albeit chronic, infection: diagnosis and treatment. Clin Microbiol Rev 2001; 14: 727-752.

7. Tezal M, Nasca MS, Stoler DL, et al. Chronic periodontitis - human papillomavirus synergy in base of tongue cancers. Arch Otolaryngol Head Neck Surg 2009; 135: 391-396.
8. Burt B; Research, Science and Therapy Committee of the American Academy of Periodontology. Position paper: epidemiology of periodontal diseases. J Periodontol 2005; 76: 1406-1419.

9. Tezal M. Interaction between chronic inflammation and oral HPV infection in the etiology of head and neck cancers. Int J Otolaryngol 2012; 2012: 575242.

10. Tezal M, Sullivan MA, Hyland A, et al. Chronic periodontitis and the incidence of head and neck squamous cell carcinoma. Cancer Epidemiol Biomarkers Prev 2009; 18: 2406-2412.

11. Lax AJ, Thomas W. How bacteria could cause cancer: one step at a time. Trends Microbiol 2002; 10: 293-299.

12. Karin M, Lawrence T, Nizet V. Innate immunity gone awry: linking microbial infections to chronic inflammation and cancer. Cell 2006; 124: 823-835.

13. Huycke MM, Gaskin HR. Commensal bacteria, redox stress, and colorectal cancer: mechanisms and models. Exp Biol Med 2004; 229: 586-597.

14. Homann N, Tillonen J, Rintamäki H, Salaspuro M, Lindqvist C, Meurman JH. Poor dental status increases acetaldehyde production from ethanol in saliva: a possible link to increased oral cancer risk among heavy drinkers. Oral Oncol 2001; 37: 153-158.

15. Visapää JP, Götte K, Benesovaetal M. Increased cancer risk in heavy drinkers with the alcohol dehydrogenase $1 C^{\star} 1$ allele, possibly due to salivary acetaldehyde. Gut 2004; 53: 871-876.

16. Salaspuro MP. Acetaldehyde, microbes, and cancer of the digestive tract. Crit Rev Clin Lab Sci 2003; 40: 183-208.

17. Shapiro KP, Hotchkiss JH, Roe DA. Quantitative relationship between oral nitrate-reducing activity and the endogenous formation of N-nitrosoamino acids in humans. Food Chem Toxicol 1991; 29: 751-755.

18. Mirvish SS. Role of N-nitroso compounds (NOC) and N-nitrosation in etiology of gastric, esophageal, nasopharyngeal and bladder cancer and contribution to cancer of known exposures to NOC. Cancer Lett 1995; 93: 17-48.

19. Rüegg C. Leukocytes, inflammation and angiogenesis in cancer: fatal attractions. J Leukoc Biol 2006; 80: 682-684.

20. Asting AG, Carén H, Andersson M, Lönnroth C, Lagerstedt K, Lundholm K. COX2 gene expression in colon cancer tissue related to regulating factors and promoter methylation status. BMC Cancer 2011; 11: 238.

21. Grivennikov SI, Karin M. Inflammatory cytokines in cancer: tumour necrosis factor and interleukin 6 take the stage. Ann Rheum Dis 2011; 70 Suppl 1: i104-i108.

22. Tatemichi M, Ogura T, Esumi H. Impact of inducible nitric oxide synthase gene on tumor progression. Eur J Cancer Prev 2009; 18: 1-8.

23. Allen C, Duffy S, Teknosetal T. Nuclear factor-kappaB-related serum factors as longitudinal biomarkers of response and survival in advanced oropharyngeal carcinoma. Clin Cancer Res 2007; 13: 3182-3190.

24. Greten FR, Eckmann L, Greten TF, et al. IKKbeta links inflammation and tumorigenesis in a mouse model of colitis-associated cancer. Cell 2004; 118: 285-296.

25. Wang D, Wang H, Shi Q, et al. Prostaglandin E2 promotes colorectal adenoma growth via transactivation of the nuclear peroxisome proliferator-activated receptor delta. Cancer Cell 2004; 6: 285-295.

26. Stetler-Stevenson WG. The tumor microenvironment: regulation by MMP-independent effects of tissue inhibitor of metalloproteinases-2. Cancer Metastasis Rev 2008; 27: 57-66.

27. Leaner VD, Donninger H, Birrer MJ. Transcription factors as targets for cancer therapy: AP-1 a potential therapeutic target. Curr Cancer Ther Rev 2007; 3: 1-6.

28. Sparmann A, Bar-Sagi D. Ras-induced interleukin-8 expression plays a critical role in tumor growth and angiogenesis. Cancer Cell 2004; 6: 447-458. 
29. Dauer DJ, Ferraro B, Songetal L. Stat3 regulates genes common to both wound healing and cancer. Oncogene 2005; 24: 3397-3408.

30. Cassatella MA, Huber V, Calzetti F, et al. Interferon-activated neutrophils store a TNF-related apoptosis-inducing ligand (TRAIL/ Apo-2 ligand) intracellular pool that is readily mobilizable following exposure to proinflammatory mediators. J Leukoc Biol 2006; 79: 123-132.

31. Williams DA. Inflammatory cytokines and mucosal injury. J Natl Cancer Inst Monogr 2001; 29: 26-30.

32. Pöllänen MT, Salonen JI, Uitto VJ. Structure and function of the tooth-epithelial interface in health and disease. Periodontology 2000; 31: 12-31.

33. Han YW, Houcken W, Loos BG, Schenkein HA, Tezal M. Periodontal disease, atherosclerosis, adverse pregnancy outcomes, and head-and-neck cancer. Adv Dent Res 2014; 26: 47-55. 